\title{
Trajectory Optimization using the Reduced Eccentric Anomaly Low-Thrust Coefficients
}

\author{
Jennifer S. Hudson * \\ University of Michigan, Ann Arbor, MI
}

\author{
Daniel J. Scheeres ${ }^{\dagger}$ \\ University of Colorado, Boulder, $C O$
}

\begin{abstract}
A method to evaluate the trajectory dynamics of low-thrust spacecraft is refined and applied to targeting and optimal control problems. The original method uses averaged variational equations for the osculating orbital elements with 14 Fourier coefficients of the thrust acceleration vector to estimate a spacecraft trajectory over many spiral orbits. The accuracy of this method is improved by correction of any offset of the averaged trajectory from the true trajectory due to non-trivial periodic components. Spacecraft targeting problems are then solved using the corrected averaged variational equations and a general cost function represented as a Fourier series. A method for reducing the cost of a transfer by selection of acceleration Fourier coefficients beyond the 14 that appear in the averaged equations is described.
\end{abstract}

\section{Introduction}

Low-thrust propulsion systems offer an efficient option for many interplanetary and Earth orbit missions. However, optimal control of these systems can pose a difficult design challenge. Analytical or approximate solutions exist for several special cases of optimal low-thrust orbit transfer problems, but the general continuous-thrust problem requires full numerical integration of each initial condition and thrust profile. The trajectory is highly sensitive to these variables, thus the optimal control law over tens or hundreds of orbits of a spiral trajectory is often difficult to determine.

Classical analytical methods exist for certain special case transfers, such as the logarithmic spiral ${ }^{1}$ and Lawden's spiral. ${ }^{2}$ More recent solutions have used the calculus of variations ${ }^{3}$ or direct optimization methods ${ }^{4}$ to determine optimal low-thrust control laws within certain constraints. Several methods for open-loop, minimum-time transfers ${ }^{5-7}$ and optimal transfers using Lyapunov feedback control ${ }^{8,9}$ also exist. Averaging methods, in combination with other approaches, have proven effective in overcoming sensitivities to small variations in initial orbit and thrust profile. ${ }^{10-12}$ Yet all solutions remain limited to certain regions of the thrust and orbital parameter space.

Previous work ${ }^{13}$ has established the validity of an averaging method to estimate the trajectory dynamics of low-thrust spacecraft in the general case. The spacecraft thrust vector components are represented as Fourier series in eccentric anomaly and the Gauss variational equations are averaged over one orbit to define a set of secular equations. These equations are functions of only 14 of the thrust Fourier coefficients, regardless of the order of the original Fourier series, and can represent thrust controls of varying magnitude and direction. An iterative method using these averaged secular equations was shown to solve orbital targeting problems with significantly reduced computing requirements as compared to integration of the full Newtonian equations of motion.

The current study further develops this method to improve the accuracy of the averaging and targeting methods. A corrective term is included in the averaging method to eliminate offsets due to periodic variations of the trajectory. This correction reduces divergence between the true and average trajectories over many orbits.

The original targeting method is generalized to accommodate any cost function represented as a Fourier series. For most cost functions, some numerical evaluation of partial derivatives is required, however the

*PhD Candidate, Department of Aerospace Engineering, University of Michigan, Ann Arbor, MI 48019

${ }^{\dagger}$ A. Richard Seebass Professor, Aerospace Engineering Sciences, Colorado Center for Astrodynamics Research, University of Colorado, Boulder, CO 80309, Member AAS, Associate Fellow AIAA 
minimum-energy cost function can be evaluated analytically. The targeting algorithm is also improved by offset correction of the initial orbital elements.

After the 14 Fourier coefficients for a given transfer have been selected, the total energy of the transfer can often be reduced by inclusion of additional terms in the thrust acceleration Fourier series. The existence of these additional energy-reduction terms is proven for certain cases and a method for selection of the additional terms is described.

\section{Applications}

This trajectory analysis and targeting method has applications to many problems of interest. It can allow designers in the early stages of mission planning to quickly perform first-order analyses of many trajectories using any thrust-based criteria of interest. Representation of the control law by its influential and noninfluential Fourier coefficients allows clear visualization of the effects of changes to the thrust profile. It can also reveal means by which an orbit transfer cost may be reduced without significantly altering the trajectory, through the method described in Section B or similar techniques.

The analytical methods described in this paper also have applications to space situational awareness, in both trajectory prediction and reverse engineering. Given limited observation data, space agencies must be able to expeditiously predict whether an unknown object or errant spacecraft poses a threat to a critical asset. In other situations, the orbital history of a known or suspected low-thrust spacecraft must be traced based on discrete sightings. The averaged variational equations in Section III may be integrated forward or backwards in time to accomplish either objective.

As the space environment becomes increasingly cluttered, the computational complexity of trajectory prediction algorithms becomes crucial. Likewise, as low-thrust propulsion systems are used in more spacecraft, predictive tools must be equipped to deal with their unique dynamics. The methods described in this paper are well suited for dealing with both high volumes of data and more detailed analyses of specific scenarios.

\section{Averaged Variational Equations}

We consider a spacecraft of negligible mass in orbit about a central body, which is assumed to be a point mass. The spacecraft is subject to a continuous thrust acceleration of potentially varying magnitude and direction. The spacecraft trajectory can be described by the Newtonian equations of motion:

$$
\begin{aligned}
\dot{\overrightarrow{\mathbf{r}}} & =\overrightarrow{\mathbf{v}} \\
\dot{\overrightarrow{\mathbf{v}}} & =-\frac{\mu}{\mathbf{r}^{3}} \overrightarrow{\mathbf{r}}+\overrightarrow{\mathbf{F}}
\end{aligned}
$$

where $\overrightarrow{\mathbf{r}}$ is the position vector, $\overrightarrow{\mathbf{v}}$ is the velocity vector, and $\mu$ is the standard gravitational parameter of the central body. The thrust acceleration vector $\overrightarrow{\mathbf{F}}$ can be resolved along the radial, normal, and tangential directions:

$$
\overrightarrow{\mathbf{F}}=F_{R} \hat{\mathbf{r}}+F_{W} \hat{\mathbf{w}}+F_{S}(\hat{\mathbf{w}} \times \hat{\mathbf{r}})
$$

where $\hat{\mathbf{r}}=\frac{\overrightarrow{\mathbf{r}}}{|\overrightarrow{\mathbf{r}}|}$ and $\hat{\mathbf{w}}=\frac{\overrightarrow{\mathbf{r}} \times \overrightarrow{\mathbf{v}}}{|\overrightarrow{\mathbf{r}} \times \overrightarrow{\mathbf{v}}|}$. The Newtonian equations can be decomposed into the Lagrange Planetary Equations, which describe the time rate of change of the classical orbit elements of a body subject to the $F_{R}, F_{W}$, and $F_{S}$ perturbations. The Gauss form of the Lagrange Planetary Equations is presented below: ${ }^{14}$

$$
\begin{aligned}
\frac{d a}{d t} & =2 \sqrt{\frac{a}{\mu}}\left[F_{R} \frac{a e}{\sqrt{1-e^{2}}} \sin \nu+F_{S} \frac{a^{2} \sqrt{1-e^{2}}}{a(1-e \cos E)}\right] \\
\frac{d e}{d t} & =\sqrt{\frac{a}{\mu}} \sqrt{1-e^{2}}\left[F_{R} \sin \nu+F_{S}(\cos \nu+\cos E)\right] \\
\frac{d i}{d t} & =\sqrt{\frac{a}{\mu}} \frac{(1-e \cos E)}{\sqrt{1-e^{2}}} F_{W} \cos (\nu+\omega) \\
\frac{d \Omega}{d t} & =\sqrt{\frac{a}{\mu}} \frac{(1-e \cos E)}{\sqrt{1-e^{2}}} F_{W} \sin (\nu+\omega)
\end{aligned}
$$




$$
\begin{aligned}
\frac{d \omega}{d t} & =\sqrt{\frac{a}{\mu}} \frac{\sqrt{1-e^{2}}}{e}\left[-F_{R} \cos \nu+F_{S}\left(1+\frac{1-e \cos E}{1-e^{2}}\right) \sin \nu\right]-\cos i \frac{d \Omega}{d t} \\
\frac{d \epsilon_{1}}{d t} & =-2 \sqrt{\frac{a}{\mu}}(1-e \cos E) F_{R}+\left(1-\sqrt{1-e^{2}}\right)(\dot{\omega}+\dot{\Omega})+2 \sqrt{1-e^{2}} \sin ^{2}\left(\frac{i}{2}\right) \dot{\Omega}
\end{aligned}
$$

In these equations, $a$ is the semi-major axis, $e$ is the eccentricity, $i$ is the inclination, $\Omega$ is the longitude of the ascending node, $\omega$ is the argument of periapsis, and $\epsilon_{1}+\int n d t=l$ is the mean longitude. The mean anomaly is the difference of the mean longitude and the longitude of periapsis:

$$
M=\int n d t+\epsilon_{1}-(\Omega+\omega)
$$

In the modeling and simulation of low-thrust spacecraft orbits, both the Newtonian equations and the Gauss equations provide identical results. The Gauss equations are often preferred for clear visualization of the orbit over time.

Given an arbitrary acceleration vector $\overrightarrow{\mathbf{F}}$, each component can be represented as a Fourier series over an arbitrary time interval $(0, \mathrm{~L})$. The Fourier series can be expanded in time or in a time-varying orbital parameter, such as true anomaly, eccentric anomaly, or mean anomaly. As shown in the previous paper, ${ }^{13}$ expansion in eccentric anomaly leads to useful simplifications.

$$
\begin{aligned}
& F_{R}=\sum_{k=0}^{\infty}\left[\alpha_{k}^{R} \cos \left(\frac{2 \pi k E}{L}\right)+\beta_{k}^{R} \sin \left(\frac{2 \pi k E}{L}\right)\right] \\
& F_{W}=\sum_{k=0}^{\infty}\left[\alpha_{k}^{W} \cos \left(\frac{2 \pi k E}{L}\right)+\beta_{k}^{W} \sin \left(\frac{2 \pi k E}{L}\right)\right] \\
& F_{S}=\sum_{k=0}^{\infty}\left[\alpha_{k}^{S} \cos \left(\frac{2 \pi k E}{L}\right)+\beta_{k}^{S} \sin \left(\frac{2 \pi k E}{L}\right)\right]
\end{aligned}
$$

We assume an acceleration vector that is specified over one orbit period $(L=2 \pi)$ with a sufficiently low magnitude that the size and shape of the orbit does not change significantly over one revolution. Therefore we may average the Gauss equations over one orbit period with respect to mean anomaly, then shift the independent parameter of the averaging to eccentric anomaly to correspond to the acceleration Fourier series, to find equations for the mean orbit elements. The average rates of change of the orbital elements $a, e, i, \Omega$, $\omega$, and $\epsilon_{1}$ are only dependent on the 14 Fourier coefficients $\alpha_{0}^{R}, \alpha_{1}^{R}, \alpha_{2}^{R}, \beta_{1}^{R}, \alpha_{0}^{S}, \alpha_{1}^{S}, \alpha_{2}^{S}, \beta_{1}^{S}, \beta_{2}^{S}, \alpha_{0}^{W}, \alpha_{1}^{W}$, $\alpha_{2}^{W}, \beta_{1}^{W}$, and $\beta_{2}^{W}$, regardless of the order of the original thrust Fourier series.

$$
\begin{aligned}
\dot{\dot{a}}= & 2 \sqrt{\frac{a^{3}}{\mu}}\left[\frac{1}{2} e \beta_{1}^{R}+\sqrt{1-e^{2}} \alpha_{0}^{S}\right] \\
\overline{\dot{e}}= & \sqrt{\frac{a}{\mu}} \sqrt{1-e^{2}}\left[\frac{1}{2} \sqrt{1-e^{2}} \beta_{1}^{R}+\alpha_{1}^{S}-\frac{3}{2} e \alpha_{0}^{S}-\frac{1}{4} e \alpha_{2}^{S}\right] \\
\overline{\dot{i}}= & \sqrt{\frac{a}{\mu}} \frac{1}{\sqrt{1-e^{2}}}\left[\frac{1}{2}\left(1+e^{2}\right) \cos \omega \alpha_{1}^{W}-\frac{3}{2} e \cos \omega \alpha_{0}^{W}-\frac{1}{2} \sqrt{1-e^{2}} \sin \omega \beta_{1}^{W}\right. \\
& \left.-\frac{1}{4} e \cos \omega \alpha_{2}^{W}+\frac{1}{4} e \sqrt{1-e^{2}} \sin \omega \beta_{2}^{W}\right] \\
\overline{\dot{\Omega}}= & \sqrt{\frac{a}{\mu}} \frac{\csc i}{\sqrt{1-e^{2}}}\left[\frac{1}{2} \sqrt{1-e^{2}} \cos \omega \beta_{1}^{W}+\frac{1}{2}\left(1+e^{2}\right) \sin \omega \alpha_{1}^{W}-\frac{3}{2} e \sin \omega \alpha_{0}^{W}\right. \\
& \left.-\frac{1}{4} e \sqrt{1-e^{2}} \cos \omega \beta_{2}^{W}-\frac{1}{4} e \sin \omega \alpha_{2}^{W}\right] \\
\overline{\dot{\omega}}= & \sqrt{\frac{a}{\mu}} \frac{1}{e}\left[-\frac{1}{2} \sqrt{1-e^{2}} \alpha_{1}^{R}+e \sqrt{1-e^{2}} \alpha_{0}^{R}+\frac{1}{2}\left(2-e^{2}\right) \beta_{1}^{S}-\frac{1}{4} e \beta_{2}^{S}\right]-\cos i \dot{\Omega} \\
\overline{\epsilon_{1}}= & \sqrt{\frac{a}{\mu}}\left[\left(-2-e^{2}\right) \alpha_{0}^{R}+2 e \alpha_{1}^{R}-\frac{1}{2} e^{2} \alpha_{2}^{R}\right]+
\end{aligned}
$$




$$
\left(1-\sqrt{1-e^{2}}\right)(\overline{\dot{\omega}}+\bar{\Omega})+2 \sqrt{1-e^{2}} \sin ^{2}\left(\frac{i}{2}\right) \dot{\Omega}
$$

The assumption of a thrust acceleration vector specified over only one orbit period is not strictly necessary; the same averaging method can be used with acceleration functions specified over arbitrary lengths, simply by averaging the Gauss equations over the full interval. In general, when $L=m \pi$, the 0 th, $\frac{m}{2}$-th, and $\mathrm{m}$-th coefficients will remain, with fractional indices required in the original Fourier series when $m$ is not an even integer. However, the averaging assumption may become less valid for aperiodic control laws of long duration, for which the orbit changes significantly from start to finish.

\section{Agreement with Newtonian Equations}

To verify the averaged secular equations, we first consider a simple control law: a step acceleration function in the tangential direction only, as pictured in Figure 1.

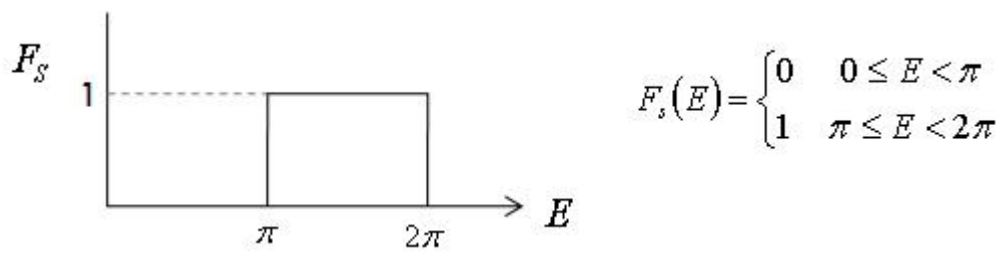

Figure 1. Step Tangential Acceleration

The Fourier series for this step function is defined by only a constant term and the odd-numbered sine coefficients:

$$
\begin{aligned}
F_{S} & =\sum_{k=0}^{\infty} \alpha_{k}^{S} \cos (k E)+\beta_{k}^{S} \sin (k E) \\
\alpha_{0}^{S} & =\frac{1}{2 \pi} \int_{0}^{2 \pi} F_{S} d E=\frac{1}{2} \\
\alpha_{k}^{S} & =\frac{1}{\pi} \int_{0}^{2 \pi} F_{S} \cos (k E) d E=0 \\
\beta_{k}^{S} & =\frac{1}{\pi} \int_{0}^{2 \pi} F_{S} \sin (k E) d E=\frac{(-1)^{k}-1}{k \pi}
\end{aligned}
$$

Figure 2 compares this Fourier series, numerically evaulated up to order 1000, to the series of only the five terms that appear in the averaged secular equations. Clearly there is considerable variation between these two representations of the periodic step acceleration control law. 


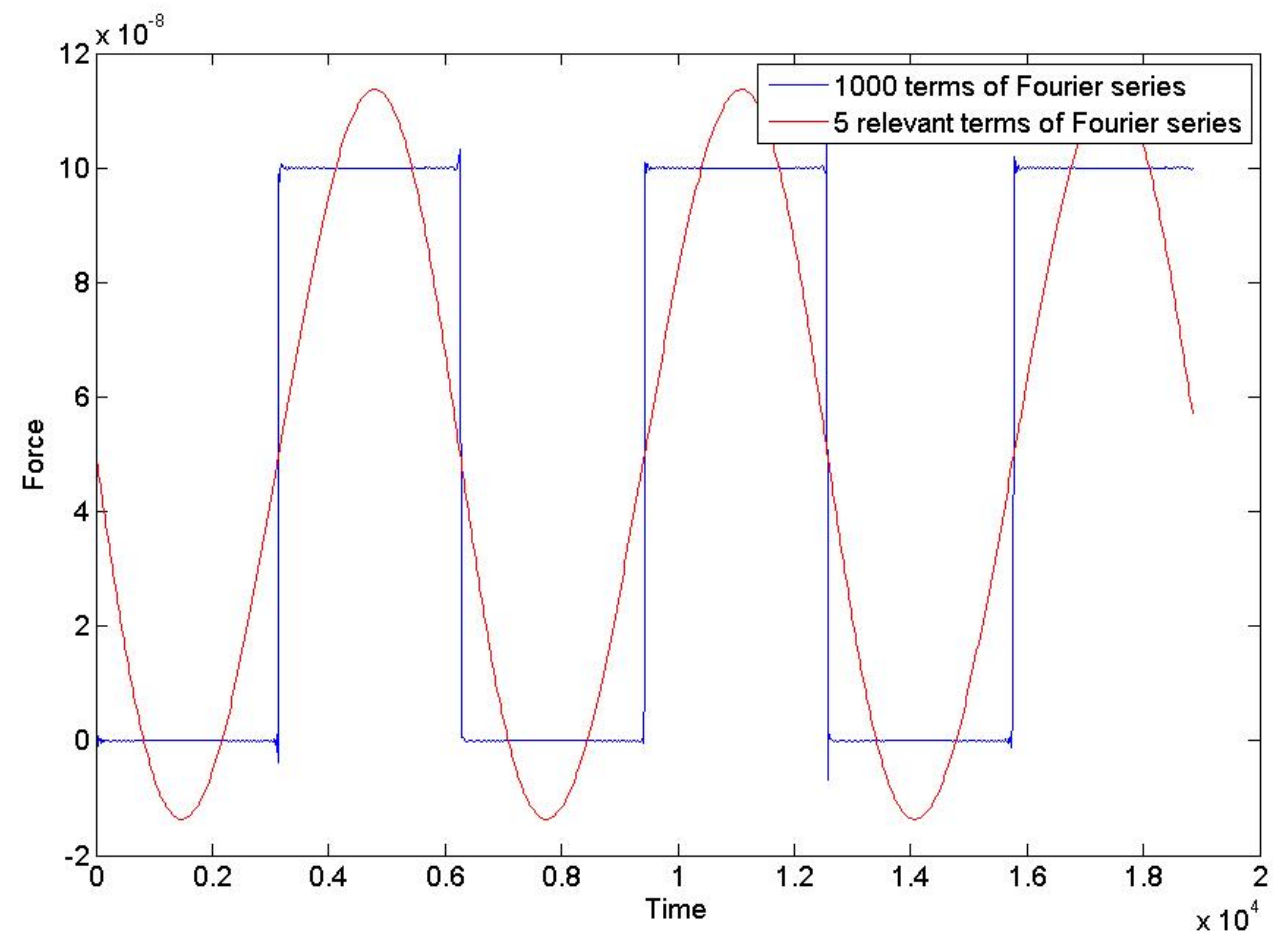

Figure 2. Step Tangential Acceleration: Comparison of thrust laws from Fourier series of step function up to order 1000 and five terms of thrust law that appear in averaged secular equations 
Figure 3 describes the osculating orbital elements of an example spacecraft subjected to these thrusts. Equations 14 - 19 with $\alpha_{0}^{S}, \alpha_{1}^{S}, \alpha_{2}^{S}, \beta_{1}^{S}$, and $\beta_{2}^{S}$ above were integrated using a Runge-Kutta method to estimate the trajectory over 10 orbits. For comparison, the Newtonian equations (Equations 1 and 2) were also integrated using the Fourier series up to order 1000. The two methods determined very similar orbital trajectories. (All dimensions herein are normalized to a standard gravitational parameter $\mu=1$.)
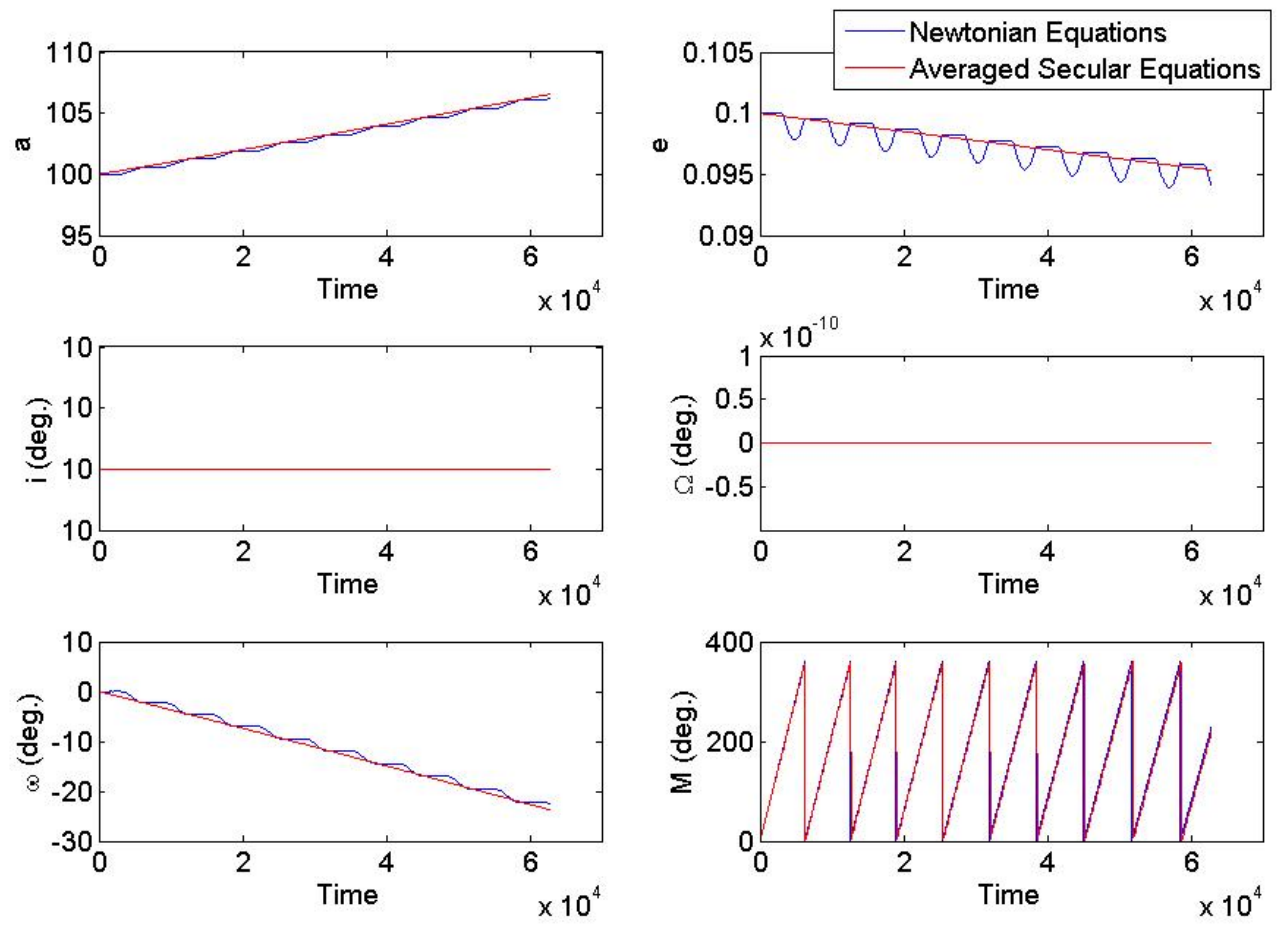

Figure 3. Step Tangential Acceleration: Comparison of trajectories determined by integration of Newtonian equations with Fourier series up to order 1000 and secular equations with five Fourier coefficients 


\section{Offset Correction}

Average trajectories calculated using Equations 14-19 show the correct trends in the evolution of the osculating orbital elements and are good approximations of the true trajectories. However, they may be offset from the true averages and may diverge from true trajectories over many orbits. This may be partially due to higher order effects not captured in the averaging method, but it may also be due to non-trivial periodic components which can shift the mean value of the state from the initial condition. This initial condition offset can be corrected by the addition of an averaged periodic term to the initial conditions of the secular equation for each orbital element.

At any time $t$, the true value of any orbital element can be expressed as the sum of a secular term and a periodic term. The periodic term repeats itself over each orbit.

$$
œ(t)=œ_{0}+\dot{\bar{œ}} t+œ_{p}(t)
$$

The true average value of the orbital element over one orbit is thus:

$$
\bar{\propto}=œ_{0}+\frac{T}{2} \dot{\bar{ळ}}+\bar{ळ}_{p}
$$

where T represents the period, $\vec{x}$ represents a vector of the six orbital elements, and $\dot{\bar{\propto}}=\bar{f}(\vec{x})$ represents Equations 14-19.

The time derivative of Equation 24 provides a differential equation for the periodic term:

$$
\dot{\propto}_{p}=f(\vec{x}, t)-\bar{f}(\vec{x})
$$

We substitute the nominal inital condition $\vec{x}_{0}$ for the true orbital element vector, knowing that the corrections are of higher order, and perform the quadrature for $œ_{p}$ :

$$
œ_{p}=\int_{0}^{t} f\left(\vec{x}_{0}, \tau\right) d \tau-\bar{f}\left(\overrightarrow{x_{0}}\right) t
$$

This periodic term can be averaged over one orbit:

$$
\bar{\propto}_{p}=\frac{1}{2 \pi} \int_{0}^{2 \pi}\left[\int_{0}^{t} f\left(\vec{x}_{0}, \tau\right) d \tau-\bar{f}\left(\vec{x}_{0}\right) t\right] d M
$$

For compatibility with the form of the Gauss equations above, the two integrals are shifted to eccentric anomaly.

$$
\bar{ळ}_{p}=\frac{1}{2 \pi} \int_{0}^{2 \pi}\left[\int_{E_{0}}^{E} \frac{1}{n}\left(1-e \cos E^{\prime}\right) f\left(\overrightarrow{x_{0}}, E^{\prime}\right) d E^{\prime}\right](1-e \cos E) d E-\frac{\pi}{n} \bar{f}\left(\overrightarrow{x_{0}}\right)
$$

where $n=\sqrt{\frac{\mu}{a^{3}}}$ is the mean motion. Note that $\frac{\pi}{n}=\frac{T}{2}$. This expression for $\bar{œ}_{p}$ can be substituted into Equation 25 to determine the average value of the orbital element over the first period.

$$
\bar{\propto}=œ_{0}+\frac{1}{2 \pi} \int_{0}^{2 \pi}\left[\int_{E_{0}}^{E} \sqrt{\frac{a^{3}}{\mu}}\left(1-e \cos E^{\prime}\right) f\left(\overrightarrow{x_{0}}, E^{\prime}\right) d E^{\prime}\right](1-e \cos E) d E
$$

To correct the initial conditions for the averaged secular equations, Equation 29 is substituted into Equation 24 at $t=0$. The averaged secular equations, thus initialized, yield a more accurate average of the true periodic trajectory.

$$
œ(0)=œ_{0}+\frac{1}{2 \pi} \int_{0}^{2 \pi}\left[\int_{E_{0}}^{E} \frac{1}{n}\left(1-e \cos E^{\prime}\right) f\left(\vec{x}, E^{\prime}\right) d E^{\prime}\right](1-e \cos E) d E-\frac{T}{2} \bar{f}(\vec{x})
$$

To calculate a value for the averaged periodic correction term, we substitute the Fourier series in eccentric anomaly for the acceleration component terms in the Gauss equations. To avoid infinite series in the solution, we only include the 14 terms of the Fourier series whose coefficients appear in the averaged secular equations, 
as they have been shown to have the most significant effect on the trajectory dynamics. Assuming zero eccentric anomaly initial conditions, the correction terms are:

$$
\begin{aligned}
& \bar{a}_{p}=\frac{a^{3}}{\mu}\left[e\left((2+e) \alpha_{0}^{R}+\frac{1}{2} \alpha_{1}^{R}-\left(\frac{1}{2} e+\frac{2}{3}\right) \alpha_{2}^{R}+\pi \beta_{1}^{R}\right)+\sqrt{1-e^{2}}\left(2 \pi \alpha_{0}^{S}+(2+e) \beta_{1}^{S}+\beta_{2}^{S}\right)\right] \\
& -\frac{T}{2} \overline{\dot{a}}\left(\vec{x}_{0}\right) \\
& \bar{e}_{p}=\frac{1}{2} \frac{a^{2}}{\mu}\left[\left(1-e^{2}\right)\left((2+e) \alpha_{0}^{R}+\frac{1}{2} \alpha_{1}^{R}-\left(\frac{1}{2} e+\frac{2}{3}\right) \alpha_{2}^{R}+\pi \beta_{1}^{R}\right)+\sqrt{1-e^{2}}\left(-3 e \pi \alpha_{0}^{S}+2 \pi \alpha_{1}^{S}\right.\right. \\
& \left.\left.-\frac{1}{2} e \pi \alpha_{2}^{S}+\left(1-\frac{5}{4} e^{2}-\frac{8}{3} e\right) \beta_{1}^{S}+\left(-\frac{5}{8} e+\frac{8}{3}\right) \beta_{2}^{S}\right)\right]-\frac{T}{2} \bar{e}\left(\vec{x}_{0}\right) \\
& \bar{i}_{p}=\frac{1}{2} \frac{a^{2}}{\mu} \frac{1}{\sqrt{1-e^{2}}}\left[\alpha_{0}^{W}\left(\sqrt{1-e^{2}} \sin \omega\left(-1-\frac{1}{2} e\right)-3 \pi e \cos \omega\right)+\alpha_{1}^{W}\left(\pi \cos \omega\left(1+e^{2}\right)\right.\right. \\
& \left.+\sqrt{1-e^{2}} \sin \omega\left(\frac{2}{3} e+\frac{1}{4} e^{2} \frac{1}{2}\right)\right)+\alpha_{2}^{W}\left(\sqrt{1-e^{2}} \sin \omega\left(\frac{2}{3}+\frac{5}{8} e\right)-\frac{1}{2} \pi e \cos \omega\right) \\
& \beta_{1}^{W}\left(\cos \omega\left(\frac{1}{2}-\frac{8}{3} e-\frac{3}{4} e^{2}\right)-\pi \sqrt{1-e^{2}} \sin \omega\right)+\beta_{2}^{W}\left(\cos \omega\left(\frac{4}{3}-\frac{9}{8} e+\frac{4}{3} e^{2}+\frac{1}{2} e^{3}\right)\right. \\
& \left.\left.+\frac{1}{2} \pi e \sqrt{1-e^{2}} \sin \omega\right)\right]-\frac{T}{2} \bar{i}\left(\vec{x}_{0}\right) \\
& \bar{\Omega}_{p}=\frac{1}{2} \frac{a^{2}}{\mu} \frac{\csc i}{\sqrt{1-e^{2}}}\left[\operatorname { c o s } \omega \sqrt { 1 - e ^ { 2 } } \left(\alpha_{0}^{W}\left(2+\frac{1}{2} e\right)+\alpha_{1}^{W}\left(-\frac{1}{4} e^{2}+\frac{1}{2}-\frac{2}{3} e\right)\right.\right. \\
& \left.+\alpha_{2}^{W}\left(-\frac{5}{8} e-\frac{2}{3}\right)+\pi \beta_{1}^{W}-\frac{1}{2} e \pi \beta_{2}^{W}\right)+\sin \omega\left(\pi \alpha_{1}^{W}\left(1+e^{2}\right)-\frac{7}{2} \pi e \alpha_{2}^{W}+\beta_{1}^{W}\left(\frac{1}{2}-\frac{8}{3} e-\frac{3}{4} e^{2}\right)\right. \\
& \left.\left.+\beta_{2}^{W}\left(\frac{4}{3}-\frac{9}{8} e+\frac{4}{3} e^{2}+\frac{1}{2} e^{3}\right)\right)\right]-\frac{T}{2} \overline{\dot{\Omega}}\left(\vec{x}_{0}\right) \\
& \bar{\omega}_{p}=\frac{1}{2} \frac{a^{2}}{\mu} \frac{1}{e}\left[\alpha_{0}^{S}\left(4-\frac{3}{2} e-2 e^{2}-e^{3}\right)+\alpha_{1}^{S}\left(\pi-\frac{2}{3} e-\frac{3}{4} e^{2}\right)+\alpha_{2}^{S}\left(-\frac{4}{3}-\frac{9}{8} e+\frac{2}{3} e^{2}+\frac{1}{2} e^{3}\right)\right. \\
& \left.\pi \beta_{1}^{S}\left(2-e^{2}\right)-\frac{1}{2} \pi \beta_{2}^{S}+2 e \pi \sqrt{1-e^{2}} \alpha_{0}^{R}-\pi \sqrt{1-e^{2}} \alpha_{1}^{R}+\beta_{1}^{R} \sqrt{1-e^{2}}\left(-\frac{1}{2}+2 e+e^{2}\right)\right] \\
& -\cos i \bar{\Omega}_{p}+\frac{T}{2} \bar{\Omega}\left(\vec{x}_{0}\right)-\frac{T}{2} \overline{\dot{\omega}}\left(\vec{x}_{0}\right) \\
& \bar{\epsilon}_{1}=-\frac{a^{2}}{\mu}\left[\pi \alpha_{0}^{R}\left(2+e^{2}\right)-2 \pi e \alpha_{1}^{R}+\frac{1}{2} \pi e^{2} \alpha_{2}^{R}+\beta_{1}^{R}\left(2-e+\frac{2}{3} e^{2}\right)\right]+\left(1-\sqrt{1-e^{2}}\right)\left(\omega_{p}+\Omega_{p}\right. \\
& \left.+\frac{T}{2} \overline{\dot{\omega}}\left(\vec{x}_{0}\right)+\frac{T}{2} \overline{\dot{\Omega}}\left(\vec{x}_{0}\right)\right)+2 \sqrt{1-e^{2}} \sin ^{2} \frac{i}{2}\left(\Omega_{p}+\frac{T}{2} \dot{\Omega}\left(\vec{x}_{0}\right)\right)-\frac{T}{2} \bar{\epsilon}_{1}\left(\vec{x}_{0}\right)
\end{aligned}
$$

Figure 4 shows the effect of these corrections on the system with Fourier coefficients randomly selected up to order 10 within the range $(-2.5 \mathrm{e}-7,2.5 \mathrm{e}-7)$. The corrected initial conditions shift the estimated average trajectory to more accurately reflect the true average trajectory. They also can reduce the divergence between the true and average trajectories, most noticeably in the plot of mean anomaly. Some drift remains, however, due to higher order effects. This can be seen in the plot of the argument of periapsis; the corrected trajectory closely approximates the true average trajectory over the first few orbits, but diverges as higher order effects accumulate over several orbits.

\section{Targeting}

The averaged secular variational equations provide an efficient means of solving low-thrust orbit transfer problems. Any spacecraft targeting problem with a time span sufficient to allow low-thrust transfer can theoretically be solved by numerical integration of the averaged equations and iterative evaluation of the 14 thrust Fourier coefficients.

In the previous paper ${ }^{13}$ an iterative method was developed to solve targeting problems using a minimumenergy cost function. This method can be extended to more general minimization problems by representing 

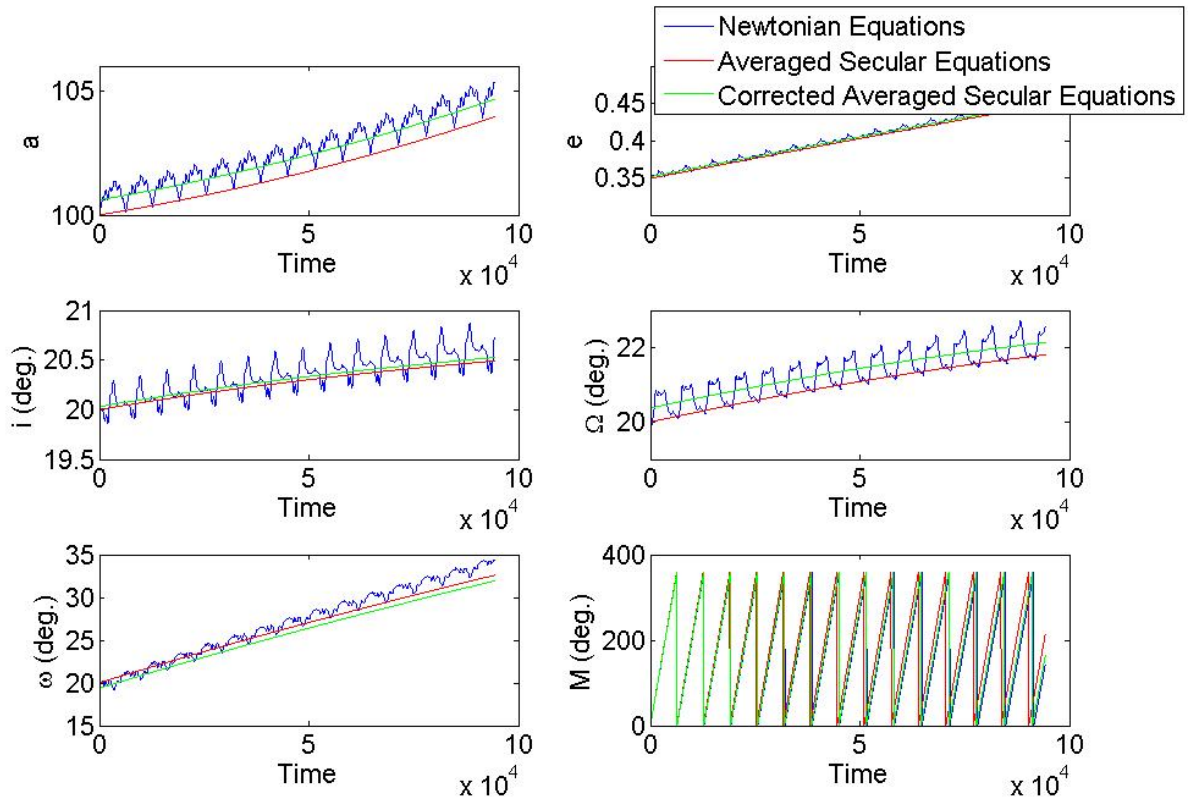

Figure 4. Averaged Trajectory Offset Correction

the cost function as the integral of another Fourier series in eccentric anomaly. Most cost functions require numerical evaluation of certain partial derivatives in order to be used in this manner, thus the minimumenergy cost function, which can be evaluated analytically, remains the most straightforward example. The targeting algorithm can also be improved by correction of the initial orbital elements according to the method described in Section V.

After a solution to the targeting problem has been found, the total energy of the transfer can often be reduced by including additional terms in the force Fourier series. Additional terms beyond the 14 that appear in the secular equations do not significantly alter the trajectory, but can, in many cases, be selected to provide a lower-cost control.

\section{A. Targeting in the Averaged Equations}

We approach the targeting problem in the averaged equations as a two point boundary value problem with given initial state $\overrightarrow{x_{0}}$, final state $\overrightarrow{x_{f}}$, and transfer time $T$. The equations of motion have the form:

$$
\dot{\vec{x}}=G(\vec{x}) \vec{\alpha}+F(\vec{x})
$$

where $\vec{x}$ is the state vector of orbital elements, $\vec{\alpha}$ is the $14 \times 1$ vector of thrust Fourier coefficients that appear in the averaged secular equations, and $\vec{F}$ accounts for the additional mean motion term in the differential equation for mean anomaly. The term $G(\vec{x}) \vec{\alpha}$ represents Equations $14-19$. 


$$
\vec{x}=\left[\begin{array}{c}
a \\
e \\
i \\
\Omega \\
\omega \\
M
\end{array}\right], \quad \vec{\alpha}=\left[\begin{array}{c}
\alpha_{0}^{R} \\
\alpha_{1}^{R} \\
\alpha_{2}^{R} \\
\beta_{1}^{R} \\
\alpha_{0}^{S} \\
\alpha_{1}^{S} \\
\alpha_{2}^{S} \\
\beta_{1}^{S} \\
\beta_{2}^{S} \\
\alpha_{0}^{W} \\
\alpha_{1}^{W} \\
\alpha_{2}^{W} \\
\beta_{1}^{W} \\
\beta_{2}^{W}
\end{array}\right], \quad \vec{F}=\left[\begin{array}{l}
0 \\
0 \\
0 \\
0 \\
0 \\
n
\end{array}\right]
$$

We minimize the cost function $J(\vec{\alpha})$, representing the minimum solution, subject to $\vec{x}\left(T ; \vec{\alpha}, \overrightarrow{x_{0}}\right)=\overrightarrow{x_{f}}$. To solve this problem, we introduce the Lagrange multiplier $\vec{\lambda}$ :

$$
\begin{array}{r}
\bar{J}=J(\vec{\alpha})+\vec{\lambda}\left[\vec{x}\left(T ; \vec{\alpha}, \overrightarrow{x_{0}}\right)-\overrightarrow{x_{f}}\right] \\
\frac{\partial \bar{J}}{\partial(\vec{\alpha}, \vec{\lambda})}=[\mathbf{0}]_{20 x 1}=\left[\begin{array}{c}
\frac{\partial J}{\partial \vec{\alpha}}+\vec{\lambda} \frac{\partial \vec{x}}{\partial \vec{\alpha}} \\
\vec{x}\left(T ; \vec{\alpha}, \overrightarrow{x_{0}}\right)-\overrightarrow{x_{f}}
\end{array}\right]
\end{array}
$$

We replace $\vec{x}\left(T ; \vec{\alpha}, \overrightarrow{x_{0}}\right)$ with its Taylor series approximation:

$$
\vec{x}\left(T ; \vec{\alpha}, \overrightarrow{x_{0}}\right) \approx \vec{x}\left(T ; \overrightarrow{\alpha_{0}}, \overrightarrow{x_{0}}\right)+\frac{\partial \vec{x}}{\partial \vec{\alpha}} \cdot \delta \vec{\alpha}
$$

For clarity, let $\frac{\partial \vec{x}}{\partial \vec{\alpha}}=\Psi_{6 \times 14}$. Beginning with the zero thrust case, we iteratively update the thrust coefficient vector $\vec{\alpha}=\overrightarrow{\alpha_{0}}+\delta \vec{\alpha}$ by solving the two simultaneous vector equations from (40).

$$
\begin{array}{r}
\left.\frac{\partial J}{\partial \vec{\alpha}}\right|_{\vec{\alpha}+\delta \vec{\alpha}}+\vec{\lambda} \cdot \Psi=0 \\
\vec{x}\left(T ; \overrightarrow{\alpha_{0}}, \overrightarrow{x_{0}}\right)+\Psi \cdot \delta \vec{\alpha}-\overrightarrow{x_{f}}=0
\end{array}
$$

We assume a cost function of the form:

$$
J(\vec{\alpha})=\frac{1}{2 \pi} \int_{0}^{2 \pi} f(\overrightarrow{\mathbf{F}}) d M
$$

where $\overrightarrow{\mathbf{F}}$ represents the force due only to the 14 relevant terms in the component Fourier series. If the integrand is represented as a Fourier series:

$$
f(\overrightarrow{\mathbf{F}})=\sum_{j=0}^{\infty} a_{j} \cos (j E)+b_{j} \sin (j E)
$$

and the independent variable of the integration is shifted to eccentric anomaly, only the first two terms of the Fourier series remain: 


$$
\begin{aligned}
J(\vec{\alpha}) & =\frac{1}{2 \pi} \int_{0}^{2 \pi}\left[\sum_{j=0}^{\infty} a_{j} \cos (j E)+b_{j} \sin (j E)\right](1-e \cos E) d E \\
& =a_{0}-\frac{e}{2} a_{1}
\end{aligned}
$$

By the definition of Fourier coefficients:

$$
\begin{aligned}
& a_{0}=\frac{1}{2 \pi} \int_{0}^{2 \pi} f(\overrightarrow{\mathbf{F}}) d E \\
& a_{1}=\frac{1}{2 \pi} \int_{0}^{2 \pi} f(\overrightarrow{\mathbf{F}}) \cos (E) d E
\end{aligned}
$$

To evaluate Equation 42, we need the partial derivative $\frac{\partial J(\vec{\alpha})}{\partial \vec{\alpha}}$, which has the form:

$$
\frac{\partial J(\vec{\alpha})}{\partial \vec{\alpha}}=\frac{\partial a_{0}}{\partial \vec{\alpha}}-\frac{e}{2} \frac{\partial a_{1}}{\partial \vec{\alpha}}
$$

This partial derivative can be evaluated numerically for most cost functions and may be evaluated analytically for certain simple cost functions.

First, we consider the cost function $J(\vec{\alpha})$ representing the minimum acceleration solution to the targeting problem. We assume a negligible rate of mass loss such that this is simply the average thrust per orbit.

$$
J(\vec{\alpha})=\frac{1}{2 \pi} \int_{0}^{2 \pi} \sqrt{F_{R}^{2}+F_{S}^{2}+F_{W}^{2}} d M
$$

Equations 47 and 48 become:

$$
\begin{aligned}
& a_{0}=\frac{1}{2 \pi} \int_{0}^{2 \pi} \sqrt{F_{R}^{2}+F_{S}^{2}+F_{W}^{2}} d E \\
& a_{1}=\frac{1}{2 \pi} \int_{0}^{2 \pi} \sqrt{F_{R}^{2}+F_{S}^{2}+F_{W}^{2}} \cos (E) d E
\end{aligned}
$$

However, the the partial derivatives of these coefficients are difficult to evaluate analytically and are undefined in the zero thrust case:

$$
\begin{aligned}
& \frac{\partial a_{0}}{\partial \vec{\alpha}}=\frac{1}{4 \pi} \int_{0}^{2 \pi} \frac{\frac{\partial}{\partial \vec{\alpha}}\left(F_{R}^{2}+F_{S}^{2}+F_{W}^{2}\right)}{\sqrt{F_{R}^{2}+F_{S}^{2}+F_{W}^{2}}} d E \\
& \frac{\partial a_{0}}{\partial \vec{\alpha}}=\frac{1}{4 \pi} \int_{0}^{2 \pi} \frac{\frac{\partial}{\partial \vec{\alpha}}\left(F_{R}^{2}+F_{S}^{2}+F_{W}^{2}\right) \cos (j E)}{\sqrt{F_{R}^{2}+F_{S}^{2}+F_{W}^{2}}} d E
\end{aligned}
$$

To avoid this problem, we consider a slightly different cost function $J(\vec{\alpha})$ representing the minimum energy solution:

$$
J(\vec{\alpha})=\frac{1}{2 \pi} \int_{0}^{2 \pi}\left(F_{R}^{2}+F_{S}^{2}+F_{W}^{2}\right) d M
$$

The orthogonality conditions lead to a simple expression for Equation 47. To simplify Equation 48, we note that the square of each force vector component is a finite sum of products of two elements of $\vec{\alpha}$ and two sines or cosines of $i E$, where $i=0,1$ or 2 . Using trigonometric identities, each product can be represented as 
a sum of sines and cosines of $j E$, where $j=0,1,2,3$ or 4 . Thus the orthogonality conditons can be applied again.

$$
\begin{aligned}
& a_{0}=\frac{1}{2}\left(\vec{\alpha} \cdot \vec{\alpha}+\left(\alpha_{0}^{R}\right)^{2}+\left(\alpha_{0}^{S}\right)^{2}+\left(\alpha_{0}^{W}\right)^{2}\right) \\
& a_{1}=2 \alpha_{0}^{R} \alpha_{1}^{R}+\alpha_{1}^{R} \alpha_{2}^{R}+2 \alpha_{0}^{S} \alpha_{1}^{S}+\alpha_{1}^{S} \alpha_{2}^{S}+\beta_{1}^{S} \beta_{2}^{S}+2 \alpha_{0}^{W} \alpha_{1}^{W}+\alpha_{1}^{W} \alpha_{2}^{W}+\beta_{1}^{W} \beta_{2}^{W}
\end{aligned}
$$

Now the partial derivative $\frac{\partial J(\vec{\alpha})}{\partial \vec{\alpha}}$ is straightforward to evaulate analytically:

$$
\frac{\partial J(\vec{\alpha})}{\partial \vec{\alpha}}=\vec{\alpha}\left(Q-\frac{1}{2} e Z\right)
$$

where

$$
\begin{aligned}
& Q=\left[\begin{array}{ccc}
q_{4} & 0 & 0 \\
0 & q_{5} & 0 \\
0 & 0 & q_{5}
\end{array}\right]_{14 \times 14}, q_{4}=\left[\begin{array}{cccc}
2 & 0 & 0 & 0 \\
0 & 1 & 0 & 0 \\
0 & 0 & 1 & 0 \\
0 & 0 & 0 & 1
\end{array}\right], q_{5}=\left[\begin{array}{ccccc}
2 & 0 & 0 & 0 & 0 \\
0 & 1 & 0 & 0 & 0 \\
0 & 0 & 1 & 0 & 0 \\
0 & 0 & 0 & 1 & 0 \\
0 & 0 & 0 & 0 & 1
\end{array}\right] \\
& Z=\left[\begin{array}{ccc}
z_{4} & 0 & 0 \\
0 & z_{5} & 0 \\
0 & 0 & z_{5}
\end{array}\right]_{14 \times 14}, z_{4}=\left[\begin{array}{cccc}
0 & 2 & 0 & 0 \\
2 & 0 & 1 & 0 \\
0 & 1 & 0 & 0 \\
0 & 0 & 0 & 0
\end{array}\right], z_{5}=\left[\begin{array}{lllll}
0 & 2 & 0 & 0 & 0 \\
2 & 0 & 1 & 0 & 0 \\
0 & 1 & 0 & 0 & 0 \\
0 & 0 & 0 & 0 & 1 \\
0 & 0 & 0 & 1 & 0
\end{array}\right]
\end{aligned}
$$

Equations 42 and 43 can now be solved to iteratively update $\vec{\alpha}$ :

$$
\begin{aligned}
\delta \vec{\alpha} & =-\left(Q-\frac{1}{2} e Z\right)^{-T} \Psi^{T} \lambda^{T}-\overrightarrow{\alpha_{0}} \\
\lambda^{T} & =\left[\Psi\left(Q-\frac{1}{2} e Z\right)^{-T} \Psi^{T}\right]^{-1}\left(\vec{x}\left(T ; \overrightarrow{\alpha^{\prime}}, \overrightarrow{x_{0}}\right)-\overrightarrow{x_{f}}-\Psi \overrightarrow{\alpha_{0}}\right)
\end{aligned}
$$

To evaluate the matrix $\Psi$, we take the partial derivative of Equation 38 with respect to $\vec{\alpha}$.

$$
\frac{\partial}{\partial \vec{\alpha}}(\dot{\vec{x}})=\left[\frac{\partial \vec{F}}{\partial \vec{x}}+\vec{\alpha} \frac{\partial G}{\partial \vec{x}}\right]\left(\frac{\partial \vec{x}}{\partial \vec{\alpha}}\right)+G(\vec{x})
$$

Ignoring the $\vec{\alpha} \frac{\partial G}{\partial \vec{x}}$ term, which will be small compared to $\frac{\partial \vec{F}}{\partial \vec{x}}$, we have an equation of the form:

$$
\begin{aligned}
& \dot{\Psi}(t)=\frac{\partial \vec{F}(t)}{\partial \vec{x}} \Psi(t)+G(\vec{x}(t)) \\
& \Psi(0)=0
\end{aligned}
$$

We note that the matrix $\frac{\partial \vec{F}}{\partial \vec{x}}$ has only one nonzero element. The solution to the homogeneous system is the orbital element transition matrix $\Phi=\frac{\partial \vec{x}}{\partial \overrightarrow{x_{0}}}$. 


$$
\begin{aligned}
& \dot{\Psi}_{h}=\left[\begin{array}{cccccc}
0 & 0 & 0 & 0 & 0 & 0 \\
0 & 0 & 0 & 0 & 0 & 0 \\
0 & 0 & 0 & 0 & 0 & 0 \\
0 & 0 & 0 & 0 & 0 & 0 \\
0 & 0 & 0 & 0 & 0 & 0 \\
-\frac{3}{2} \frac{n}{a} & 0 & 0 & 0 & 0 & 0
\end{array}\right] \Psi \\
& \Psi_{h}=\left[\begin{array}{cccccc}
1 & 0 & 0 & 0 & 0 & 0 \\
0 & 1 & 0 & 0 & 0 & 0 \\
0 & 0 & 1 & 0 & 0 & 0 \\
0 & 0 & 0 & 1 & 0 & 0 \\
0 & 0 & 0 & 0 & 1 & 0 \\
-\frac{3}{2} \frac{n}{a} t & 0 & 0 & 0 & 0 & 1
\end{array}\right]=\Phi \\
& \Phi(0)=I
\end{aligned}
$$

Using the standard procedure, we find the particular solution, then sum the homogeneous and particular solutions at $t=0$ to find the general solution:

$$
\Psi(t)=\Phi(t) \int_{0}^{t} \Phi^{-1}(\tau) G(\vec{x}(\tau)) d \tau
$$

This expression for $\Psi$ allows us to iteratively update $\vec{\alpha}$ using Equation 61 and converge on a solution to the boundary value problem that minimizes the cost function.

The initial condition offset correction described in Section V improves the accuracy of the targeting algorithm. We initialize the algorithm with zero thrust, then use the Fourier coefficients calculated in each iteration to correct the initial state of the subsequent iteration according to Equation 31. Thus each integration of the averaged equations has a slightly different initial conditon which more closely approximates the average of the desired transfer trajectory.

$$
{\overrightarrow{x_{0 j+1}}}_{j}=\left[\begin{array}{c}
a(0)_{j}+a_{p}(0)_{j+1} \\
e(0)_{j}+e_{p}(0)_{j+1} \\
i(0)_{j}+i_{p}(0)_{j+1} \\
\Omega(0)_{j}+\Omega_{p}(0)_{j+1} \\
\omega(0)_{j}+\omega_{p}(0)_{j+1} \\
M(0)_{j}+M_{p}(0)_{j+1}
\end{array}\right]
$$

This method converges to a set of 14 Fourier coefficients that describe a control law that solves the targeting problem in the averaged secular equations. The true trajectory under this control law, as determined by the Newtonian equations, generally compares well to the averaged trajectory, as demonstrated in Section IV.

An example of the targeting methodology applied to a transfer in all six orbital elements is shown below. Table 1 shows the initial and final states of the boundary value problem, between which the spacecraft must transfer over five orbits. After five iterations of the targeting algorithm, initialized with the zero thrust case, the final $\vec{\alpha}$ is shown in Table 2 and the transfer trajectory is shown in Figure 5. As above, the dimensions are normalized to a standard gravitational parameter $\mu=1$. 
Table 1. Targeting Example: Initial and Target States

\begin{tabular}{lcc}
\hline & Initial State & Final State \\
$a$ & 100 & 110 \\
$e$ & 0.1 & 0.15 \\
$i$ (deg.) & 20 & 30 \\
$\Omega$ (deg.) & 20 & 30 \\
$\omega$ (deg.) & 20 & 30 \\
$M$ (deg.) & 0 & 10
\end{tabular}

Table 2. Targeting Example: Final Force Fourier Coefficients After Five Iterations

\begin{tabular}{cc}
\hline$\alpha_{0}^{R}$ & $-0.0136 \mathrm{e}-5$ \\
$\alpha_{1}^{R}$ & $0.0961 \mathrm{e}-5$ \\
$\alpha_{2}^{R}$ & $-0.0004 \mathrm{e}-5$ \\
$\beta_{1}^{R}$ & $-0.0058 \mathrm{e}-5$ \\
$\alpha_{0}^{S}$ & $0.0001 \mathrm{e}-5$ \\
$\alpha_{1}^{S}$ & $0.2534 \mathrm{e}-5$ \\
$\alpha_{2}^{S}$ & $-0.0205 \mathrm{e}-5$ \\
$\beta_{1}^{S}$ & $0.0011 \mathrm{e}-5$ \\
$\beta_{2}^{S}$ & $-0.0149 \mathrm{e}-5$ \\
$\alpha_{0}^{W}$ & $0.0143 \mathrm{e}-5$ \\
$\alpha_{1}^{W}$ & $0.0173 \mathrm{e}-5$ \\
$\alpha_{2}^{W}$ & $0.0006 \mathrm{e}-5$ \\
$\beta_{1}^{W}$ & $-0.0338 \mathrm{e}-5$ \\
$\beta_{2}^{W}$ & $-0.0010 \mathrm{e}-5$
\end{tabular}

14 of 19 

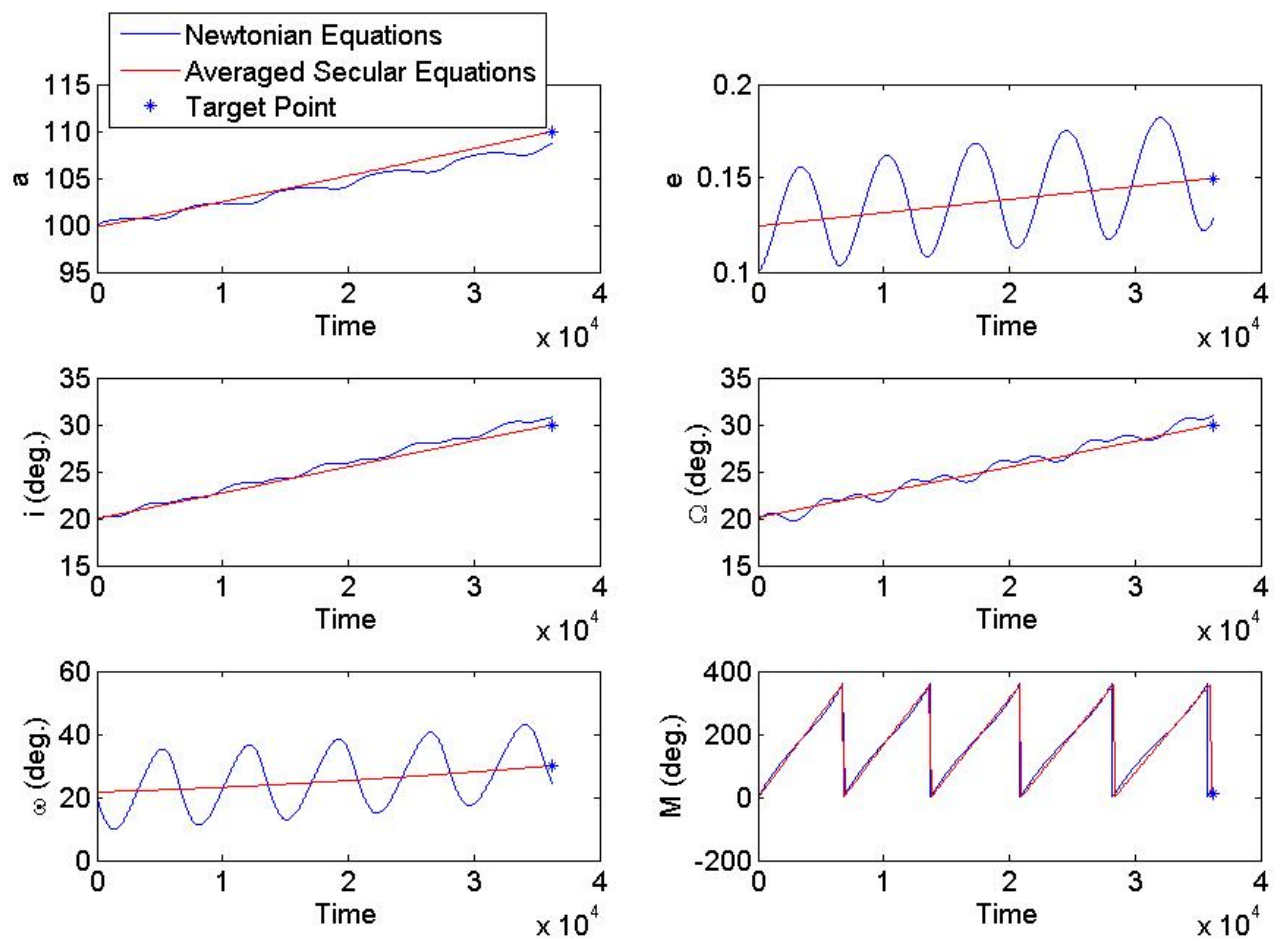

Figure 5. Targeting Example: Solution trajectory after five iterations

\section{B. Lower-Energy Transfer with Extended Thrust Fourier Series}

In many cases, additional terms in the thrust acceleration Fourier series beyond the 14 key terms can reduce the total cost of the transfer without significantly altering the trajectory. The following proves the existence of a lower-cost control for the quadratic cost function and all controls with nonzero values of $\alpha_{2}$.

The quadratic minimum energy cost function is described by Equations 46, 56, and 57. For simplicity, we consider only the cosine series of one directional component of the force:

$$
J(\vec{\alpha})=\frac{1}{2}\left[\vec{\alpha} \cdot \vec{\alpha}+\left(\alpha_{0}\right)^{2}-e\left(2 \alpha_{0} \alpha_{1}+\alpha_{1} \alpha_{2}\right)\right]
$$

If the Fourier series is extended up to order N, the difference between the new cost function and the cost function for the key-element-only series is:

$$
\Delta J=\frac{1}{2}\left[\left[\left(\alpha_{3}\right)^{2}+\left(\alpha_{4}\right)^{2}+\ldots+\left(\alpha_{N}\right)^{2}\right]-e\left(\alpha_{2} \alpha_{3}+\alpha_{3} \alpha_{4}+\ldots+\alpha_{N-1} \alpha_{N}\right)\right]
$$

If the additional terms are to reduce the energy of the transfer, we must have $\Delta J<0$. One method for this is term-by-term selection of the additional Fourier coefficients. Given a nonzero value of $\alpha_{2}$, we can choose $\alpha_{3}$ such that

$$
\alpha_{3}^{2}-e \alpha_{2} \alpha_{3}<0
$$

That is,

$$
0<\left|\alpha_{3}\right|<e\left|\alpha_{2}\right|
$$


Define $\epsilon$ such that $0<\epsilon<1$. Then $\alpha_{3}=e \epsilon \alpha_{2}$. Continuing in this manner, we can choose $\alpha_{n}$ such that

$$
\alpha_{n}=(e \epsilon)^{n-2} \alpha_{2}
$$

where $n=1 \ldots N$, so that each term further reduces $\Delta J$. Now, let $N \rightarrow \infty$ and define $J^{\prime}$ :

$$
J^{\prime}=\alpha_{2}^{2}+2 \Delta J
$$

Substituting Equations 72 and 75, we can simplify the expression:

$$
\begin{aligned}
J^{\prime} & =\sum_{j=0}^{\infty}\left[\alpha_{j+2}^{2}-e \alpha_{j+2} \alpha_{j+3}\right] \\
& =\sum_{j=0}^{\infty}\left[(e \epsilon)^{2 j}-\frac{(e \epsilon)^{2 j+2}}{\epsilon}\right] \alpha_{2}^{2} \\
& =\frac{\alpha_{2}^{2}}{1-(e \epsilon)^{2}}\left[1-e^{2} \epsilon\right]
\end{aligned}
$$

where the final simplification is based on the fact that for any $w<1, \sum_{j=0}^{\infty} w^{j}=\frac{1}{1-w}$. We can now solve Equation 76 to find the total reduction in transfer energy for a given choice of $\epsilon$ and infinite additional terms in the Fourier series:

$$
\Delta J=-\frac{1}{2} \alpha_{2}^{2} \frac{e^{2}}{1-(e \epsilon)^{2}}(1-\epsilon)
$$

Thus a lower-cost control exists for all controls with $\alpha_{2} \neq 0$. This method can be extended to select terms of the full sine and cosine series of each directional component of the force, assuming $\alpha_{2}^{R}, \beta_{1}^{R}, \alpha_{2}^{S}, \beta_{2}^{S}$,

$\alpha_{2}^{W}$, and $\beta_{2}^{W}$ are nonzero. Further methods to optimize this minimization or address other cost functions will be developed in future work.

\section{Nonsingular Equations for Circular Orbits}

In the previous paper, a change of variables was introduced to avoid singularities in the averaged secular equations due to zero eccentricity or inclination. To replace the variables $e, i, \Omega$, and $\omega$, we introduced the variables

$$
\begin{aligned}
h_{1} & =e \sin \tilde{\omega} \\
k_{1} & =e \cos \tilde{\omega} \\
h_{2} & =\sin i \sin \Omega \\
k_{2} & =\sin i \cos \Omega
\end{aligned}
$$

where $\tilde{\omega}=\Omega+\omega$. The averaged equations were reformulated in terms of these variables.

The offset correction method described in Section V can be applied to the equations in the new variables. The resulting correction terms $\bar{h}_{1 p}, \bar{k}_{1 p}, \bar{h}_{2 p}$, and $\bar{k}_{2 p}$ have the form of Equation 31. These terms are quite lengthy, however, so they are most easily calculated using a symbolic manipulator.

The variable change allows numerical integration of the averaged equations for trajectories that closely approach circular orbits. Figure 6 shows an example of the corrected equations in the new variables for an orbit originating at $e=0.005$ with Fourier coefficients randomly selected up to order 10 . 

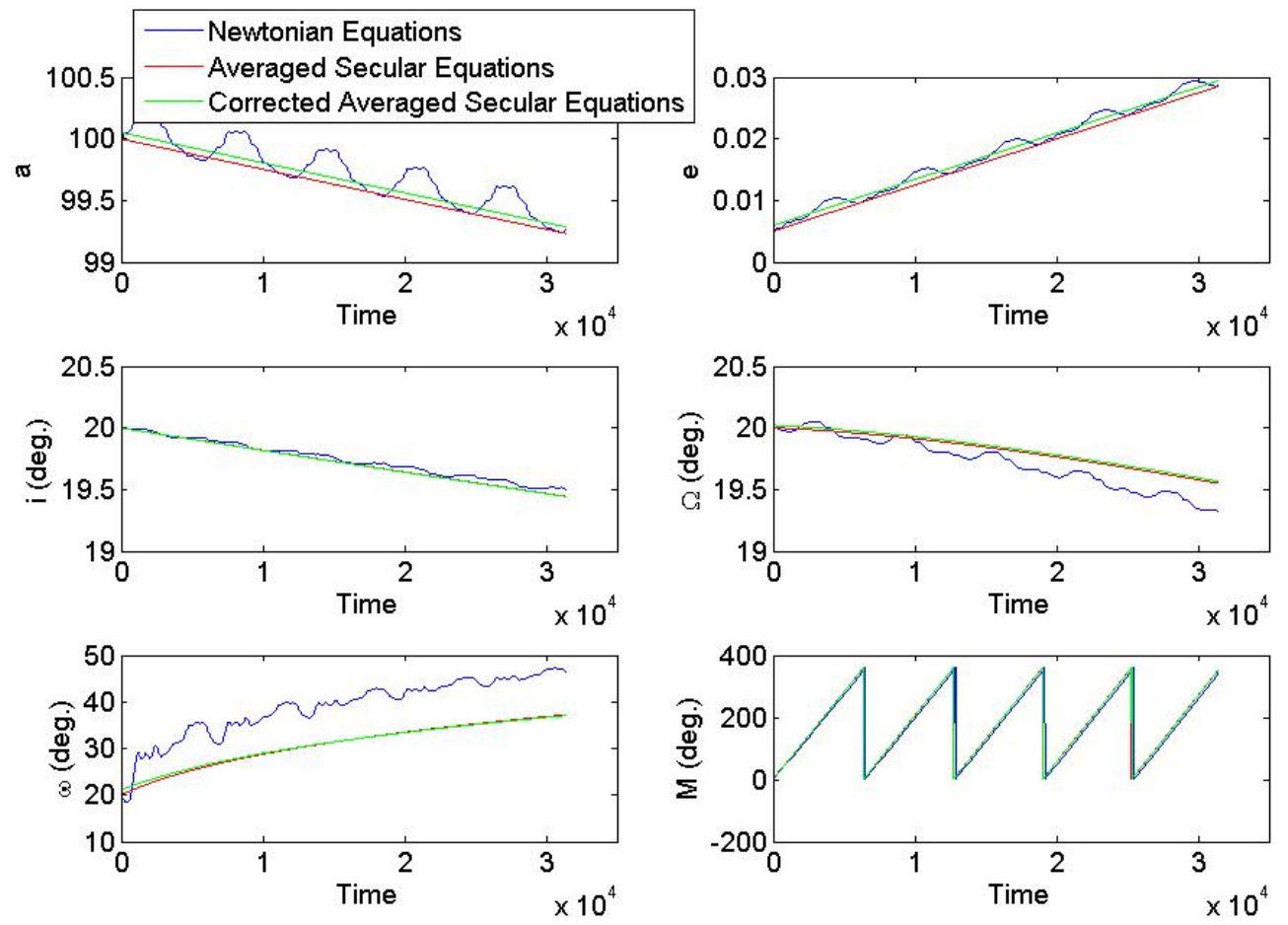

Figure 6. Trajectory initialized at $\mathrm{e}=0.005$ with corrected equations in changed variables 
Although this method is accurate for trajectories with very small eccentricities, some computational difficulties remain for trajectories involving exactly zero eccentricity. Simulations that begin from an exactly circular orbit usually do not show agreement between the averaged equations and the true trajectory. This is unlikely to be a problem in real applications, as any flight trajectory would not be exactly circular. However, it prevents comparison of this method to other low-thrust spacecraft targeting methods that involve theoretical circular orbits.

A similar situation exists for equatorial orbits, but this is of less concern, as the plane from which inclination is measured can usually be redefined.

\section{Conclusions}

The Fourier coefficient method for evaluation of average low-thrust spacecraft trajectory dynamics was refined and applied to orbital targeting problems. Offsets of the average trajectory due to periodic variations were corrected, which reduced the divergence between the true and average trajectories over several orbits. This correction, along with generalization of the cost function analysis, improved the original orbital targeting algorithm. Reduction of the energy required for a given transfer by inclusion of additional terms in the thrust Fourier series was proven possible in certain cases and a method for selection of the additional terms was described.

The improved targeting method provides accurate, low-cost solutions to almost all feasible low-thrust orbital targeting problems. It does not generally provide optimal solutions, however, as higher-order terms may always be added to the thrust acceleration vector Fourier series to reduce the total energy of the transfer, or other low-thrust solutions may exist.

Future work will explore further optimization methods using the averaged secular equations. Timevariation of the thrust acceleration vector will be included in the targeting algorithm; transfer costs may be reduced by correctly selecting different control laws for different portions of the transfer. Alternate cost functions and improved methods for selection of the additional Fourier coefficients may also be explored. Future work will also continue to investigate the remaining singularities of the circular orbit case.

\section{Acknowledgments}

This material is based upon work supported under a National Science Foundation Graduate Research Fellowship.

\section{References}

${ }^{1}$ Forbes, G.F., "The Trajectory of a Powered Rocket in Space," Journal of the British Interplanetary Society, Vol. 9, No. 2, pp. $75-79,1950$.

${ }^{2}$ Lawden, D.F., "Optimal Intermediate-Thrust Arcs in a Gravitational Field," Astronautica Acta, Vol. 8, pp. 106-123, 1962.

${ }^{3}$ Marec, J.P. and Vinh, N.X., "Optimal Low-Thrust, Limited Power Transfers between Arbitrary Elliptical Orbits," Acta Astronautica, Vol. 4, pp. 511-540, 1977.

${ }^{4}$ Kluever, C.A., and Oleson, S. R., "Direct Approach for Computing Near-Optimal Low-Thrust Earth-Orbit Transfers," Journal of Spacecraft and Rockets, Vol. 35, No. 4, pp. 509-515, 1998.

${ }^{5}$ Kluever, C.A. "Simple Guidance Scheme for Low-Thrust Orbit Transfers," Journal of Guidance, Control, and Dynamics, Vol. 21, No. 6, pp. 1015-1017, 1998.

${ }^{6}$ Kluever, C.A. "Low-Thrust Orbit Transfer Guidance Using an Inverse Dynamics Approach," Journal of Guidance, Control, and Dynamics, Vol. 18, No. 1, pp. 187-189, 1995.

${ }^{7}$ Kluever, C.A., and Shaughnessy, D. J. "Trajectory-Tracking Guidance Law for Low-Thrust Earth-Orbit Transfers," Journal of Guidance, Control, and Dynamics, Vol. 23, No. 4, pp. 754-756, 2000.

${ }^{8}$ Ilgen, M.R., Low Thrust OTV Guidance Using Lyapunov Optimal Feedback Control Techniques," AAS/AIAA Astrodynamics Specialist Conference, American Astronautical Society Paper 93-680, Aug. 1993.

${ }^{9}$ Gurfill, P., "Nonlinear Feedback Control of Low-Thrust Orbital Transfer in a Central Graviational Field," Acta Astronautica, Vol. 60, Nos. 8-9, pp. 631-648, 2007.

${ }^{10}$ Petropoulos, A. E., "Some Analytic Integrals of the Averaged Variational Equations for a Thrusting Spacecraft", IPN Progress Report 42-150, pp. 1-29, April-June 2002.

${ }^{11}$ Edelbaum, T. N., "Optimum Power-Limited Orbit Transfer in Strong Gravity Fields," AIAA Journal, Vol. 3, No. 5, pp. 921-925, May 1965.

${ }^{12}$ Gao, Y., "Low-Thrust Nonlinear Guidance by Tracking Mean Orbital Elements," Journal of Guidance, Control, and Dynmamics, Vol. 31, No. 4, pp. 1103-1110, 2008. 
${ }^{13}$ Hudson, J. and Scheeres, D. "Reduction of Low Thrust Continuous Controls for Trajectory Dynamics." 2007 AIAA/AAS Astrodynamics Specialist Conference, Aug. 2007.

${ }^{14}$ Danby, J. M. A., Fundamentals of Celestial Mechanics, 2nd ed. 5th printing, Willmann-Bell, Richmond, Virginia, pp. 327-328, 2003.

19 of 19 